ECCOMAS Proceedia
COMPDYN 2021

$8^{\text {th }}$ ECCOMAS Thematic Conference on Computational Methods in Structural Dynamics and Earthquake Engineering

M. Papadrakakis, M. Fragiadakis (eds.)

\title{
AN EQUIVALENT VISCOUS DAMPING PROPOSAL FOR BLOCK- BASED ROCKING MODELS
}

\author{
Georgios Vlachakis ${ }^{1}$, Carla Colombo ${ }^{1}$, Anastasios I. Giouvanidis ${ }^{1}$, Anjali Mehrotra ${ }^{1}$, \\ Nathanael Savalle ${ }^{1}$, and Paulo B. Lourenço ${ }^{1}$ \\ ${ }^{1}$ University of Minho, ISISE, Department of Civil Engineering, \\ 4800-058, Guimarães, Portugal
}

e-mail: giorgovlachaki@gmail.com,carla.colombo95@gmail.com, agiouvanidis@civil.uminho.pt, a.a.mehrotra@tudelft.nl,n.savalle@civil.uminho.pt,pbl@civil.uminho.pt

\begin{abstract}
Masonry structures have been observed to display a high vulnerability to failure under seismic action. This stems from the fact that their structural configurations usually lack adequate connections among the distinct elements, resulting in the formation of local mechanisms experiencing Out-Of-Plane (OOP) collapse. In this context, rocking dynamics has proven to be a valuable methodology for the analysis of masonry walls. Classical rocking theory can provide a fast solution to the dynamic phenomena taking place if simple configurations are examined. Nevertheless, as the degrees of freedom and the boundary conditions increase, the complexity increases, and thus the classical rocking theory becomes impractical. In the meantime, recent developments in computational modelling of masonry structures are gaining significant attraction. This includes block-based models which inherently consider the complexity of the problem and enable the solution to be obtained easily in the discretised spatial and time domains. However, despite their widespread use, applications of such models usually lack a reliable treatment of damping.

The present work attempts to bridge the gap between the well-established energy loss of the classical rocking theory and the treatment of damping of block-based computational models. To do so, the dynamics of the problem are reviewed and an equivalent viscous damping model is proposed. A unilateral dashpot formulation allows the replication of the impulsive nature of the energy loss at impact. Afterwards, a calibration methodology is adopted for the practical range of the problem's parameters and a ready-to-use equation is provided, which respects energy equivalence. The performance of the proposed damping model is also evaluated through comparisons with experimental results.
\end{abstract}

Keywords: rocking, coefficient of restitution, viscous damping, numerical modelling, out-ofplane. 


\section{INTRODUCTION}

Masonry structures constitute a significant part of our built heritage, with earthquakes representing a severe threat to their integrity [1]. During seismic events, such structures tend to fail via specific collapse mechanisms, with out-of-plane (OOP) collapse recognised to be the most frequent and destructive - especially in the case of façade walls inadequately connected with the rest of the structural elements [2]. Static approaches have been widely used to assess the collapse of the OOP mechanisms, resorting either to limit analysis methods [3], or to advanced numerical techniques [4]. However, static approaches display inherent limitations, since the inertial effects are neglected and the dynamic reserve of stability is disregarded. Therefore, over-conservatism characterises the static approaches [5], while dynamic techniques suggest a more reliable modelling solution [6].

In this context, rocking dynamics has been proposed to simulate the response of OOP mechanisms [7]. In the classical work of Housner [8], the equations of motion of a rigid body rocking over its base have been developed. Since then, extensive research has evaluated the influence on the rocking response of both seismological characteristics [9], and structural features, such as the presence of transverse walls $[10,11]$, horizontal elements [12-14] and restraints [13, 15-17]. Rocking dynamics assumes that energy is lost at every impact, and is captured by the Coefficient of Restitution (CoR) which correlates the angular velocity of the structure before and after impact. The CoR may be computed analytically by assuming conservation of angular momentum [8]. Despite small discrepancies, experimental campaigns have confirmed the validity of the analytical approach [18-20], particularly from a statistical perspective [21]. Nevertheless, as the Degrees-Of-Freedom (DOFs) and the BoundaryConditions (BCs) of the problem increase, the complexity introduced transforms the method to one of impractical interest [22, 23].

In the meanwhile, recent developments in numerical simulation methods with block-based models are gaining momentum, especially due to the ease in representing complex masonry textures, $\mathrm{BCs}$, interactions between the contacting bodies, and material nonlinearities. Among them, the most commonly used are the Finite Element Method (FEM) [24], the Discrete Element Method (DEM) [25], and the multi-body dynamics [26, 27]. However, despite their widespread use, block-based numerical modelling techniques lack a reliable method of replicating energy loss at impact $[28,29]$. Usually, viscous damping models are employed [30-32], yet, without confidence about their equivalence with the overall more consistent rocking theory.

The present work aims to bridge the gap between the treatment of energy loss of the statistically-accurate classical rocking theory and the widely used numerical block-based modelling. This is achieved by "tuning" a viscous damping model to replicate both the manner and extent of energy loss indicated by the rocking theory based on the CoR. To this end, the main dynamic and modelling characteristics of both the theoretical and numerical modelling techniques are described in Section 2. Subsequently, Section 3 presents the calibration methodology, resulting in the proposal of a pertinent ready-to-use equation which respects energy loss equivalence between the examined methods. Finally, the performance of the numerical viscous damping model is assessed in Section 4, where an experimental campaign from the literature is employed as a reference. 


\section{STRATEGIES FOR MODELLING ROCKING STRUCTURES}

\subsection{Analytical modelling}

Consider a rigid block exhibiting planar rocking motion over its rigid base, as shown in Figure 1 (a). In this case, the equation of motion can be written as [8]:

$$
\ddot{\theta}=-p^{2}\left[\sin ( \pm \alpha-\theta)+\frac{\ddot{u}_{g}}{g} \cos ( \pm \alpha-\theta)\right]
$$

where $\alpha$ is the slenderness of the block, $\theta$ describes the rocking response and $\ddot{u}_{g}$ denotes the seismic ground acceleration. The positive and negative signs in Eq. (1) depend on the sign of $\theta$, while $p$ is defined as $p=\sqrt{m g R / I_{0}}$, with $m$ referring to the mass of the block, and $I_{0}$ the rotational moment of inertia with respect to the pivot points. The moment-rotation diagram of the rigid system is indicated by the solid line in Figure 1 (c).

The equation of motion (Eq. (1)) describes the smooth part of the rocking motion, i.e. when pivoting, while nonsmooth impacts interrupt the response. Impacts are treated as instantaneous events that lead to energy loss in the form of radiation damping. Assuming no bouncing or sliding, the CoR $e$ sufficiently describes the contact event, by connecting the preimpact and post-impact angular velocities. Then, conservation of angular momentum yields [8]:

$$
e=1-\frac{3}{2} \sin ^{2} \alpha
$$

Clearly, the energy loss, in this case, relies solely upon the slenderness of the block and not its material characteristics.

The solution of the analytical problem herein is obtained by solving the differential Eq. (1) interrupted by event-based impacts, using mathematical programming in MATLAB.

\subsection{Numerical modelling}

Numerical block-based models introduce an interface stiffness to describe the interaction of the contact bodies (in this case, the block and the base, see Figure 1 (b)), working on the normal $\left(k_{n}\right)$ and tangential $\left(k_{s}\right)$ directions of contact. Importantly, the interface stiffness might be used to replicate actual stiffness properties of contacting bodies [33], simulate macro characteristics such as mortar flexibility [19] and degradation [34], or foundation flexibility

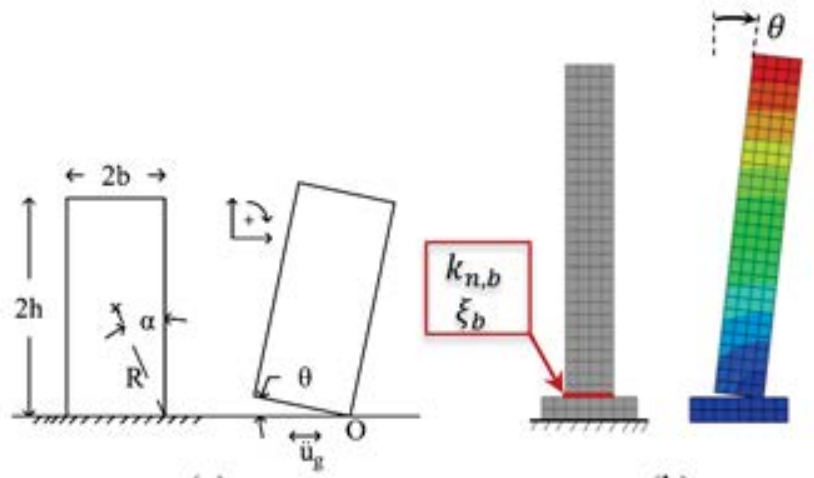

(a)

(b)

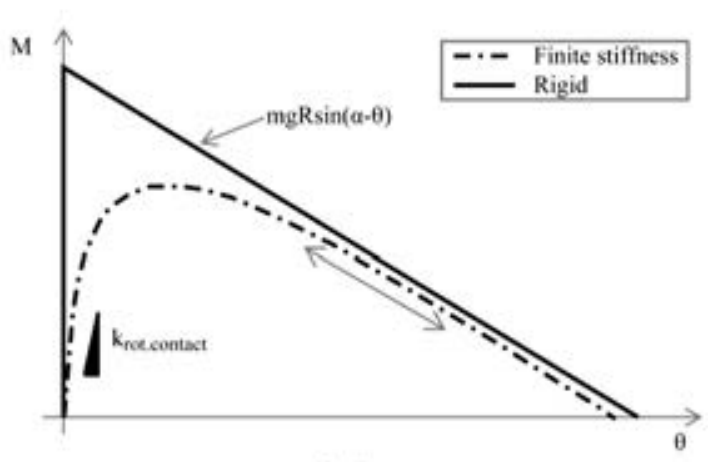

(c)

Figure 1: (a) Scheme of the classical rocking rigid block; (b) Numerical modelling of the rocking block with finite stiffness; and (c) Moment-rotation diagram of rigid and finite stiffness modelling techniques. 
[35]. Consequently, the finite stiffness of the system $\left(k_{\text {rot.contact }}\right.$, the moment-rotation curve for which is also shown also in Figure 1 (c)), results in impacts that occur over finite displacement and time, in contrast with the instantaneous behaviour of the analytical model.

Concerning energy loss, numerical block-based models commonly adopt viscous damping formulations, which are mathematical "artifices" used to simulate dissipative phenomena not considered explicitly [36]. Note that viscous damping models have a continuous nature, seemingly in contrast with the event-based CoR theory. A variety of viscous damping formulations are available depending on: i) the degrees of freedom used, ii) the response of the model during the time-history, and iii) the methods used to calibrate them [37]. Among them, a stiffness-proportional dashpot definition acting at the contact interfaces seems the most appropriate for simulation of the instantaneous features of the rocking impacts [38].

In the present work, the finite element environment of ABAQUS CAE [39] is used, where the solution is obtained using an explicit time-stepping integration. The rocking block is set to interact with its base through the normal $k_{n}$ and tangential $k_{s}$ interface stiffnesses. Moreover, a unilateral dashpot viscous damping model governs the interacting bodies, with a damping ratio $\xi$. It is worth noting that the validity of the adopted modelling technique has been confirmed also in other block-based simulation software [38], including implicit FEM and explicit DEM methods. This indicates universal applicability of the proposed numerical viscous damping model.

\section{A VISCOUS DAMPING MODEL PROPOSAL}

\subsection{Calibration methodology}

Since all viscous damping models need to be "tuned" to reproduce the desired energy dissipation, a phenomenological calibration methodology is adopted. More specifically, the energy loss of the classic rocking dynamics is considered as a reference, and the numerical viscous damping model is adjusted to mimic the former. In essence, the objective is to find a correlation among the CoR $e$ and the damping ratio $\xi$. Evidently, the free-rocking problem is examined, where dissipation phenomena have a major impact on the response.

To this end, both modelling techniques are used to solve the rocking problem and pairs of $\xi-e$ are generated based on which response-histories match the best. This is done after comparing the rocking response of the two modelling techniques and minimising their root mean square difference. During the calibration process, all parameters possibly affecting the response are examined, i.e. i) the scale $R$, ii) the slenderness $H / B$, iii) the rocking amplitude $\theta_{0} / \alpha$, and iv) the normal interface stiffness $k_{n}$. In addition, the influence of the CoR $e$ is also varied, independently of the slenderness of the block, i.e. Eq. (2) is disregarded. This choice allows the viscous damping proposal to be adaptable to any CoR correction suggestions, based either on experimental $[10,19]$, or theoretical basis $[40,41]$. The ranges of the independent parameters that are varied during the calibration process are shown in Table 1. Among them, the scale has been found not to affect the viscous damping model, while nonsignificant influence is caused by the variations of the rocking amplitude (for more details, see [38]). Therefore, only the slenderness and the normal interface stiffness are essential to be included in the viscous damping model proposal. 


\begin{tabular}{ccc}
\hline Parameter & Range & Reference value \\
\hline Scale: $R[\mathrm{~m}]$ & $1.4-2.8$ & 2.1 \\
Slenderness: $h / b[-]$ & $4.0-15.0$ & 7.0 \\
Amplitude: $\theta_{0} / \alpha[-]$ & $0.3-0.8$ & 0.5 \\
Normal interface stiffness: $k_{n}\left[\mathrm{~N} / \mathrm{m}^{3}\right]$ & $0.5 \cdot 10^{8}-30 \cdot 10^{8}$ & $5 \cdot 10^{8}$ \\
\hline
\end{tabular}

Table 1: Independent parameters considered for calibration.

\subsection{Proposed viscous damping model}

The generated $\xi-e$ pairs of all the previously mentioned variations, 245 in total, are fitted through a multivariable nonlinear regression analysis. As a result, the following predictive equation is proposed which correlates the $\mathrm{CoR} e$ with the damping ratio of the viscous model $\xi$ :

$$
\xi_{b}=-0.000292 \cdot\left(\frac{H}{B}\right)^{0.935} \cdot k_{n}^{0.343} \cdot \ln e
$$

Note that Eq. (3) is characterised by a coefficient of determination of $R^{2}=0.978$ over the 245 data $\xi-e$ pairs, showing a noteworthy estimation capability.

To illustrate the phenomenological calibration, one exemplary case of the predictive performance of Eq. (3) is shown in Figure 2. The comparison examines a rocking block with dimensions $2 h=4.2 \mathrm{~m}$ and $2 b=0.6 \mathrm{~m}, \operatorname{CoR} e=0.97$, normal interface stiffness $k_{n}=5 \cdot 10^{8}\left[\mathrm{~N} / \mathrm{m}^{3}\right]$ and damping ratio $\xi=5.9 \%$ according to Eq. (3). More specifically, Figure 2 (a) shows the rocking response of both the numerical viscous model and the analytical CoR based model, while Figure 2 (b) illustrates the corresponding energy content of both models. Clearly, a very good agreement is observed between the two methods. Importantly, the numerical viscous model presents a step-like response similar to that of the analytical CoR-based model: significant energy is lost only at impacts, gradually dissipating the freerocking response. A small divergence is noticed only at smaller rocking amplitudes, i.e. $\theta_{0} / \alpha \leq 0.1$, which, however, are not expected to be decisive for the rocking stability of the structure, and thus assumed acceptable. Overall, the numerical viscous damping model shows a similar dissipative nature with that of the analytical CoR-based model, and the fitted relationship Eq. (3) a good predictive capability.

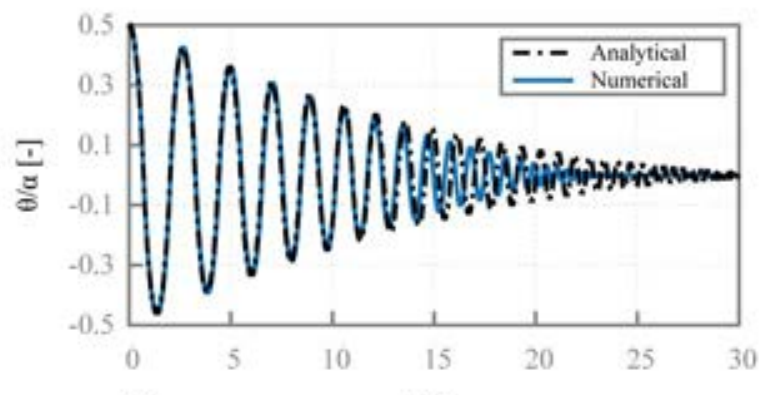

(a)

$t[s]$

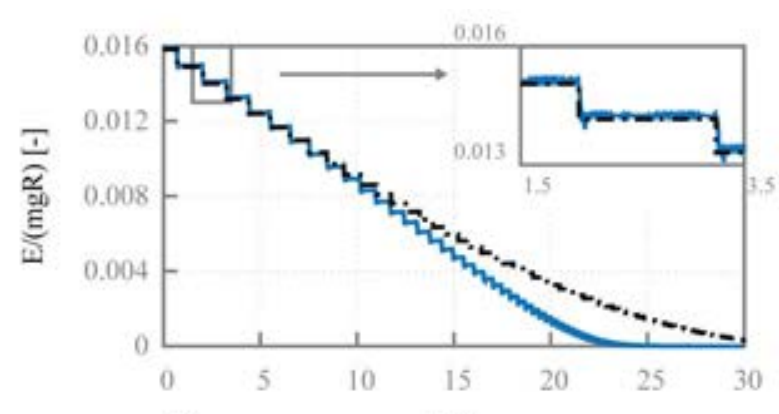

(b) $t[s]$

Figure 2: Behaviour of the viscous damping model. Comparison with the analytical model, in terms of variation of the (a) rocking angle and (b) total energy content over time. Details of the examined structure: $2 h=4.2[\mathrm{~m}]$,

$$
2 b=0.6 \mathrm{~m}, e=0.97, k_{n}=5 \cdot 10^{8}\left[\mathrm{~N} / \mathrm{m}^{3}\right] \text { and } \xi=5.9 \% \text {. }
$$




\section{EVALUATION OF THE PROPOSED VISCOUS MODEL}

Finally, the performance of the proposed numerical viscous damping model is assessed. While its calibration has been based on the analytical rocking model, its robustness is tested using actual experimental results. To this end, the experimental campaign reported by Peña [42] is used, where granite rocking blocks were tested under free- and forced-rocking vibrations. Details regarding the specimens' and excitation characteristics can be found in Table 2 and [42]. Given the fact that the proposed model has been calibrated independently of Eq. (2), the experimentally measured CoRs can be inserted in Eq. (3) to get the damping ratio $\xi$ of the viscous damping model.

\begin{tabular}{ccccc}
\hline Specimen & $2 h[\mathrm{~m}]$ & $2 b[\mathrm{~m}]$ & $e_{\exp }[-]$ & Tests examined \\
\hline 1 & 1.0 & 0.25 & 0.936 & $\begin{array}{c}\text { i) Free rocking, ii) Hanning sine with } \\
f=3.3 \mathrm{~Hz} \text { and } a_{p}=7 \mathrm{~mm}\end{array}$ \\
2 & 1.0 & 0.17 & 0.973 & i) Free rocking \\
3 & 1.0 & 0.12 & 0.978 & $\begin{array}{c}\text { i) Synthetic ground excitation record } \\
18, \text { load factor } 0.5,[42] \text { for details }\end{array}$ \\
\hline
\end{tabular}

Table 2: Details of the specimens and examined tests [42].

\subsection{Free rocking}

Figure 3 (a) compares the free-rocking response of Specimen 1, while Figure 3 (b) the freerocking response of Specimen 2, respectively. The results of the numerical simulations show a very good match with the experimental response both in terms of rocking phase and amplitude. Slight differences appear after several cycles, yet, given the experimental uncertainties and the simplicity of the problem, the overall dissipative phenomena are very well captured.

\subsection{Forced rocking}

To further evaluate the numerical viscous damping model, results of forced-rocking tests are compared in Figure 4. More specifically, Figure 4 (a) shows Specimen 1 under a hanningsine excitation, while Figure 4 (b) illustrates Specimen 3 under a synthetic ground motion excitation [42]. For both cases, the numerical viscous damping model presents a rather good estimation of the experimental response. Slight differences are also observed, most notably the earlier collapse of the numerical model of Specimen 3. Nevertheless, considering the nondeterministic and bifurcating behaviour of the rocking problem, the performance of the nu-
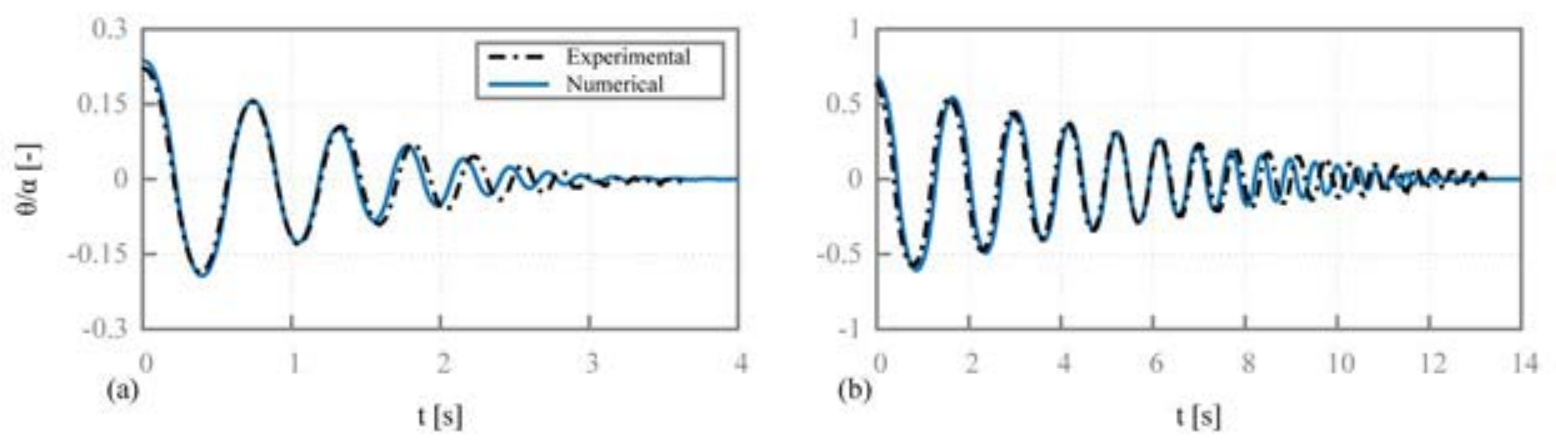

Figure 3: Free-rocking response of the proposed numerical viscous damping model compared to the experimental response reported in [42] of (a) Specimen 1 and (b) Specimen 2. 

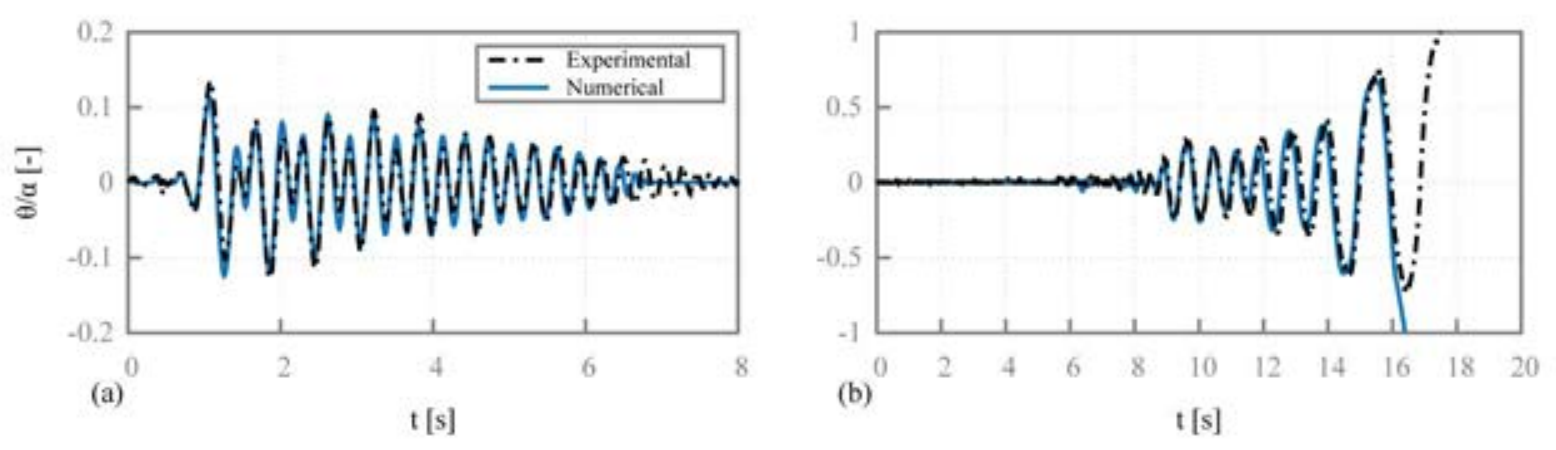

Figure 4: Forced-rocking response of the proposed numerical viscous damping model compared to the experimental response reported in [42] of (a) Specimen 1 under hanning-sine excitation and (b) Specimen 3 under synthetic ground excitation (Table 2).

merical viscous damping model is regarded as satisfactory.

\section{CONCLUSIONS}

This work proposes a numerical viscous damping model, aimed at simulation of the energy losses of the rocking problem using numerical block-based modelling techniques. This is achieved by following a phenomenological calibration methodology, where the analytical CoR-based rocking model is assumed as a reference and the viscous damping model is tuned accordingly. It is noteworthy that the suggestion of using a stiffness-proportional unilateral dashpot model results in impulsive energy losses, similarly to that of the CoR method. The performance of the proposed viscous damping model has been evaluated against experimental results from the literature, both under free- and forced-rocking vibrations, where its adequacy is highlighted.

In conclusion, the present paper suggests a reliable and consistent way to model energy losses when using numerical block-based models. This offers the possibility of modelling the rocking phenomena of more complex structures, with many DOFs or varying BCs, while additional material nonlinearities can be also easily included. Moreover, the proposed viscous damping model is universal and adaptable, as it can be used with any block-based numerical method and with any experimental or theoretical corrections on the used CoR.

\section{REFERENCES}

[1] N. Augenti and F. Parisi, "Learning from construction failures due to the 2009 L'Aquila, Italy, earthquake," J. Perform. Constr. Facil., vol. 24, no. 6, pp. 536-555, 2010.

[2] G. Vlachakis, E. Vlachaki, and P. B. Lourenço, "Learning from failure: Damage and Failure of Masonry Structures, after the 2017 Lesvos Earthquake (Greece)," Eng. Fail. Anal., vol. 117, 2020.

[3] J. Vaculik, M. C. Griffith, and G. Magenes, "Dry stone masonry walls in bending-Part II: Analysis,” Int. J. Archit. Herit., vol. 8, no. 1, pp. 29-48, 2014.

[4] G. Vlachakis, M. Cervera, G. B. Barbat, and S. Saloustros, "Out-of-plane seismic response and failure mechanism of masonry structures using finite elements with enhanced strain accuracy," Eng. Fail. Anal., vol. 97, pp. 534-555, 2019.

[5] M. Godio and K. Beyer, "Evaluation of force-based and displacement-based out-ofplane seismic assessment methods for unreinforced masonry walls through refined 
model simulations," Earthq. Eng. Struct. Dyn., vol. 48, no. 4, pp. 454-475, 2019.

[6] L. Sorrentino, D. D’Ayala, G. de Felice, M. C. Griffith, S. Lagomarsino, and G. Magenes, "Review of Out-of-Plane Seismic Assessment Techniques Applied To Existing Masonry Buildings," Int. J. Archit. Herit., vol. 11, no. 1, pp. 2-21, 2017.

[7] C. Casapulla, L. Giresini, and P. B. Lourenço, "Rocking and kinematic approaches for rigid block analysis of masonry walls: State of the art and recent developments," Buildings, vol. 7, no. 3, 2017.

[8] G. Housner, "The behavior of inverted pendulum structures during earthquakes," Bull. Seismol. Soc. Am., vol. 53, no. 2, pp. 403-417, 1963.

[9] A. I. Giouvanidis and E. G. Dimitrakopoulos, "Rocking amplification and strongmotion duration," Earthq. Eng. Struct. Dyn., vol. 47, no. 10, pp. 2094-2116, 2018.

[10] L. Sorrentino, O. AlShawa, and L. D. Decanini, "The relevance of energy damping in unreinforced masonry rocking mechanisms. Experimental and analytic investigations," Bull. Earthq. Eng., vol. 9, no. 5, pp. 1617-1642, 2011.

[11] O. Al Shawa, G. De Felice, A. Mauro, and L. Sorrentino, "Out-of-plane seismic behaviour of rocking masonry walls," Earthq. Eng. Struct. Dyn., vol. 41, no. 5, pp. 949-968, 2012.

[12] L. Giresini, M. Fragiacomo, and P. B. Lourenço, "Comparison between rocking analysis and kinematic analysis for the dynamic out-of-plane behavior of masonry walls," Earthq. Eng. Struct. Dyn., vol. 44, no. 13, pp. 2359-2376, 2015.

[13] A. Mauro, G. de Felice, and M. J. DeJong, "The relative dynamic resilience of masonry collapse mechanisms," Eng. Struct., vol. 85, pp. 182-194, 2015.

[14] O. AlShawa, D. Liberatore, and L. Sorrentino, "Dynamic One-Sided Out-Of-Plane Behavior of Unreinforced-Masonry Wall Restrained by Elasto-Plastic Tie-Rods," Int. J. Archit. Herit., vol. 13, no. 3, pp. 340-357, 2019.

[15] A. I. Giouvanidis and E. G. Dimitrakopoulos, "Seismic Performance of Rocking Frames with Flag-Shaped Hysteretic Behavior," J. Eng. Mech., vol. 143, no. 5, 2017.

[16] M. F. Vassiliou and N. Makris, "Dynamics of the Vertically Restrained Rocking Column,” J. Eng. Mech., vol. 141, no. 12, 2015.

[17] E. G. Dimitrakopoulos and A. I. Giouvanidis, "Seismic response analysis of the planar rocking frame," J. Eng. Mech., vol. 141, no. 7, p. 04015003, 2015.

[18] P. R. Lipscombe and S. Pellegrino, "Free Rocking of Prismatic Blocks," J. Eng. Mech., vol. 119, no. 7, pp. 1387-1410, 1993.

[19] A. A. Costa, A. Arêde, A. Penna, and A. Costa, "Free rocking response of a regular stone masonry wall with equivalent block approach: experimental and analytical evaluation," Earthq. Eng. Struct. Dyn., vol. 42, no. 15, pp. 2297-2319, 2013.

[20] D. Kalliontzis and S. Sritharan, "Characterizing dynamic decay of motion of freestanding rocking members," Earthq. Spectra, vol. 34, no. 2, pp. 843-866, 2018.

[21] J. A. Bachmann, M. Strand, M. F. Vassiliou, M. Broccardo, and B. Stojadinović, "Is rocking motion predictable?," Earthq. Eng. Struct. Dyn., vol. 47, no. 2, pp. 535-552, 2018. 
[22] A. I. Giouvanidis and Y. Dong, "Seismic loss and resilience assessment of single column rocking bridges," Bull. Earthq. Eng., 2020.

[23] A. Mehrotra and M. J. Dejong, "A methodology to account for interface flexibility and crushing effects in multi-block masonry collapse mechanisms," Meccanica, 2020.

[24] A. M. D'Altri et al., "Modeling Strategies for the Computational Analysis of Unreinforced Masonry Structures: Review and Classification," Arch. Comput. Methods Eng., vol. 27, pp. 1153-1185, 2019.

[25] J. V Lemos, "Discrete Element Modeling of the Seismic Behavior," Buildings, vol. 9, no. 2, 2019.

[26] A. I. Giouvanidis and E. G. Dimitrakopoulos, "Nonsmooth dynamic analysis of sticking impacts in rocking structures," Bull. Earthq. Eng., vol. 15, no. 5, pp. 22732304, 2017.

[27] F. Portioli and L. Cascini, "Contact Dynamics of Masonry Block Structures Using Mathematical Programming," J. Earthq. Eng., vol. 22, no. 1, 2018.

[28] G. de Felice, S. De Santis, P. B. Lourenço, and N. Mendes, "Methods and Challenges for the Seismic Assessment of Historic Masonry Structures," Int. J. Archit. Herit., vol. 11, no. 1, pp. 143-160, 2017.

[29] M. F. Vassiliou et al., "Shake table testing of a rocking podium : Results of a blind prediction contest," Earthq. Eng. Struct. Dyn., 2020.

[30] O. AlShawa, L. Sorrentino, and D. Liberatore, "Simulation Of Shake Table Tests on Out-of-Plane Masonry Buildings. Part (II): Combined Finite-Discrete Elements," Int. J. Archit. Herit., vol. 11, no. 1, pp. 79-93, 2017.

[31] J. V. Lemos and A. Campos Costa, "Simulation of Shake Table Tests on Out-of-Plane Masonry Buildings. Part (V): Discrete Element Approach," Int. J. Archit. Herit., vol. 11, no. 1, pp. 117-124, 2017.

[32] D. Malomo, A. Mehrotra, and M. J. Dejong, "Distinct element modeling of the dynamic response of a rocking podium tested on a shake table," Earthq. Eng. Struct. Dyn., no. December, pp. 1-7, 2020.

[33] C. Campaña, B. N. J. Persson, and M. H. Muser, "Transverse and normal interfacial stiffness of solids with randomly rough surfaces," J. Phys. Condens. Matter, vol. 23, 2011.

[34] M. C. Griffith, N. Lam, J. L. Wilson, and K. Doherty, "Experimental Investigation of Unreinforced Brick Masonry Walls in Flexure,” J. Struct. Eng., vol. 130, no. 3, 2004.

[35] P. D. Spanos, A. Di Matteo, A. Pirrotta, and M. Di Paola, "Rocking of rigid block on nonlinear flexible foundation," Int. J. Non. Linear. Mech., vol. 94, pp. 362-374, 2017.

[36] F. A. Charney, "Unintended consequences of modeling damping in structures," $J$. Struct. Eng., vol. 134, no. 4, pp. 581-592, 2008.

[37] J. F. Hall, "Problems encountered from the use (or misuse) of Rayleigh damping," Earthq. Eng. Struct. Dyn., vol. 35, no. 5, pp. 525-545, 2006.

[38] G. Vlachakis, A. I. Giouvanidis, A. Mehrotra, and P. B. Lourenço, "Numerical blockbased simulation of rocking structures using a novel universal viscous damping model," J. Eng. Mech., 2021, (under review). 
[39] Simulia, Abaqus 6.12 documentation. Rhode Island, US: Dassault Systèmes Simulia, 2012.

[40] D. Kalliontzis, S. Sritharan, and A. Schultz, "Improved Coefficient of Restitution Estimation for Free Rocking Members,” J. Struct. Eng., pp. 1-7, 2016.

[41] T. Ther and L. P. Kollar, "Refinement of Housner's model on rocking blocks," Bull. Earthq. Eng., vol. 15, pp. 2305-2319, 2017.

[42] F. Peña, P. B. Lourenço, and A. Campos-Costa, "Experimental dynamic behavior of free-standing multi-block structures under seismic loadings," J. Earthq. Eng., vol. 12, no. 6, pp. 953-979, 2008. 